American Journal of Applied Sciences 1 (1): 51-53, 2004

ISSN 1546-9239

(C) Science Publications, 2004

\title{
Standard Abundance Distribution of Elements in the Universe and Magic Numbers with Boltzmann Probability
}

\author{
${ }^{1}$ Frederick Osman and ${ }^{2}$ Heinrich Hora \\ ${ }^{1}$ School of Quantitative Methods and Mathematical Sciences, \\ University of Western Sydney, Locked Bag 1797, Penrith South DC 1797, Australia \\ ${ }^{2}$ Department of Theoretical Physics, \\ University of New South Wales, Sydney, 2052, Australia
}

\begin{abstract}
The decay of the standard abundance distribution of elements in the Universe for heavy elements has well-know maxima near magic numbers of the nuclear shell model and deep minima in between. Combining the maxima as a generation probability $N(Z)$ on the proton number $Z$ of the nuclides, a Boltzmann probability shows decay $\mathrm{N}(\mathrm{Z})=\mathrm{N}^{\prime} \exp \left(-\mathrm{Z} / \mathrm{Z}^{\prime}\right)$. The result of $\mathrm{Z}^{\prime}=10$ has consequences on the foundation of the magic numbers, on the quark structure of the shell and determines new higher magic numbers.
\end{abstract}

Key words: Magic numbers, standard abundance distribution, Boltzmann probability, Standard Abundance Distribution (SAD)

\section{INTRODUCTION}

It is well known from nuclear astrophysics that there is a Standard Abundance Distribution (SAD) of the elements in the Universe (Fig. 1) Showing for a nucleon number $A>60$ (above about irony) a nearly exponential decay of the structure of the maxims which are close to the magic numbers. Between these maxima the minima are up to 100 times and more lower. For $\mathrm{A}<60$, the abundance of the elements is very much higher resulting from the thermonuclear fission processes from protons up to iron well know from the various stages of stellar evolution.

Elements synthesis for heavier nuclides with $A>60$ cannot be due to fission because these reactions would be endothermic. Only an ensemble of nuclei with a background of neutrons may react within a Boltzmannlike equilibrium process. This changes any distribution of nuclides into the well-observed standard abundance having the exponential decaying probability for higher A or proton number $\mathrm{Z}$ of nuclei. A distribution of the abundance $\mathrm{N}(\mathrm{Z})$ depending on the proton number $\mathrm{Z}$ :

$$
N(Z)=N^{\prime} \exp \left(Z / Z^{\prime}\right)
$$

For the maxima of the SAD, drawn, as weakly bent solid line Fig. 1, for heavy nuclides is therefore rather trivial though it may be worthwhile to recognize a kind of Boltzmann equilibrium for the state of nuclides with a neutron background. Statistically there are fluctuations in nuclides until the exponential distribution has been achieved. One may assume that the occurs at an early stage of the big bang when nuclei are in some femtometer (Fermi) distance where the reaction times may be between femtoseconds and attoseconds or less.

For lower densities in supernova or in white dwarfs the endothermic element synthesis by the s-, the r- or the p-processes results in similar Boltzmann equilibrium as in Eq. 1 where, however, the reaction times are up to $10^{4}$ seconds due to the larger distances of the reacting nuclei. Similar conditions may exist in astrophysical ensembles of nuclei at similar distances and time scales if there is a proton background where the calm repulsion is compensated thermally and/or there are sufficiently high densities.

The consequences of the strong line in Fig. 1 fitting with the empirical astrophysical observations of the SAD-does result in $Z^{\prime}=10$ or values nearby. The magic numbers of the nuclear shell model are the sequence $^{[2]}$ :

Magic numbers: $\mathrm{M}_{1} \in 2,8,20,28,50,82,126$

For protons $\mathrm{Z}$ in nuclides as well as for neutrons $\mathrm{N}$ $=\mathrm{A}-\mathrm{Z}$ with the measured well-known maxima of binding energies (Fig. 2). A calculation of the ratio $R$ (n) for the astrophysical ${ }^{[1]}$ SAD-Boltzmann probabilities reflected in Eq. 1:

$\mathrm{R}(\mathrm{n})=\left[\mathrm{N}\left(\mathrm{Z}_{\mathrm{n}+1}\right) / \mathrm{N}\left(\mathrm{Z}_{\mathrm{n}}\right)\right]^{-1}=\exp \left[\left(\mathrm{Z}_{\mathrm{n}+1}-\mathrm{Z}_{\mathrm{n}}\right) / \mathrm{Z}^{\prime}\right]$

Where, the magic numbers $Z_{n}$ of the protons is taken with the following indices in $(0,1,2,3 \ldots)$ :

$\mathrm{Z}_{0}=2, \mathrm{Z}_{1}=8, \mathrm{Z}_{2}=20$

for relation up to the magic number 20

$\mathrm{Z}_{2}=28, \mathrm{Z}_{3}=50, \mathrm{Z}_{4}=82 \mathrm{Z}_{5}=126$,

for the magic numbers above 20

(Table 1) for $Z^{\prime}=10$ in (1), the ratios $R$, Eq. 3 results in values very close to (Fig. 2):

$\mathrm{R}(\mathrm{n})=3^{\mathrm{n}}$ 


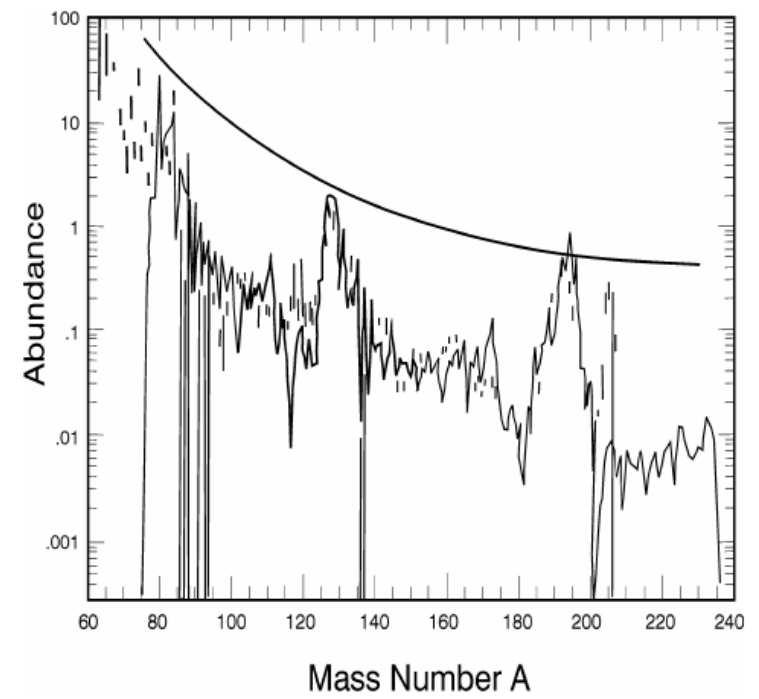

Fig. 1: The standard abundance distribution of the elements of the nucleon number A (mass number) of the Universe follows and added straight line to fit Eq. (1) ${ }^{[1]}$

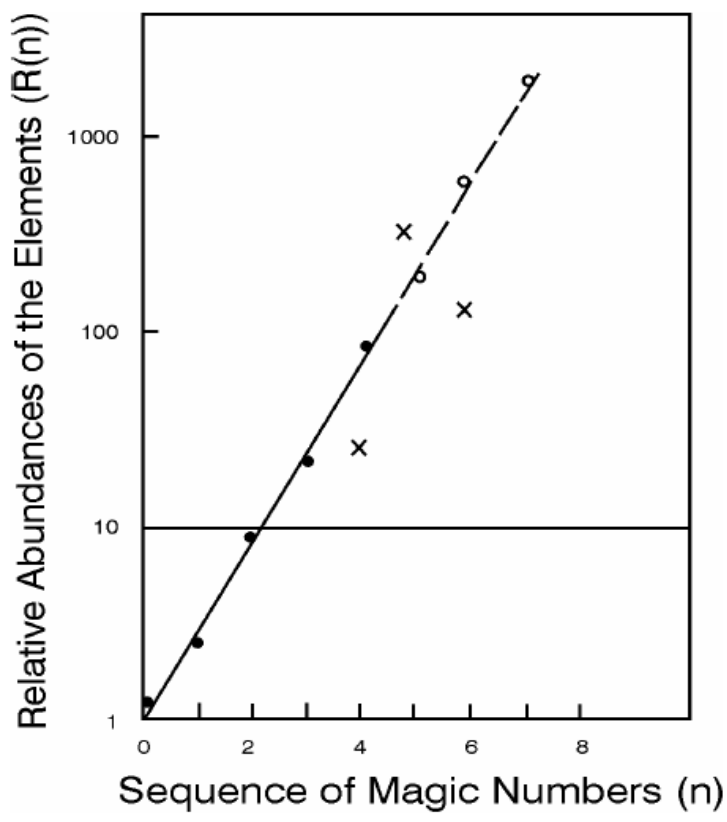

Fig. 2: Values $\left.R(n)=\exp \left[Z_{n+1}-Z_{n}\right) / Z^{\prime}\right]$ for the sequence of magic numbers $n$ with a specially defined exception of 20 and with the fitting value $Z^{\prime}=10$, (dots) compared with the $3^{\text {n }}$ - relation (Eq. 6): straight line. Circles are for the derivation of new magic numbers (180; 246 and 324), Eq. (7) and crosses for earlier considered numbers 114,184 and 228

The good fit with Z" =10 compared with other numbers can be seen in the magic numbers 82 at $n=4$. Instead of $R=81.45$ (being very close to $3^{4}$ for $Z^{\prime}=10$ ) we find $R=224.69$ for $Z^{\prime}=8 ; R=132.80$ for $Z^{\prime}=9, R=$ 54.598 for $Z^{\prime}=11$.
Extending the procedure with the $3^{\mathrm{n}}$ - law (6) to higher magic numbers-see the extension of the fully drawn line by the dashed line (Fig. 3) -one arrives at the following higher magic numbers indicated by open circles as closest values in the line. The results are that for $\mathrm{n}=6$ one arrives at a magic number 180 , for $\mathrm{n}=7$ at 246 and for $\mathrm{n}=8$ at 324 :

New magic numbers $180 ; 246 ; 324$

Shown by circles in (Fig. 2). This can be compared with the earlier predicted magic numbers 114,184 and 228 (crosses in Fig. 2) which by far do not fit ${ }^{[3]}$ so the relation (6).

The first conclusion of these results derived from this fitting of the Boltzmann probability (1) with the standard abundance distribution of the heavier elements observed in the Universe, Fig 1, refers to the curious jumping procedure with the magic numbers 20 and 28 (Table 1).

This is exactly what was necessary when the magic numbers were discovered. In order to explain the maximum binding energy of some nuclei, this could not be explained so easily as e.g. The electron shells in atoms from the Schrödinger equation with the wellknown $2 n^{2}$ - relation $(n=1,2,3 \ldots)$ for shells. It is most remarkable that a purely speculative combination of the sequences $2 ; 3 ; 4 ; 5 ; 6 \ldots$ and of the sequence $1 ; 2 ; 3 ; 4$; $5 ; \ldots$ and their second order combinations ${ }^{[4]}$ led Bagge $^{[2]}$ to the result of the following sequences:

$\mathrm{M}_{2} \in 2,8,20,40,70,112$

$\mathrm{M}_{3} \in 2,6,14,28,50,82,126$

The question was why the bold numbers fit the measurements Eq. (2) and how to explain the jump from the Bagge sequences (8) to (9) after the first three elements. The well known explanation was given ${ }^{[4]}$ by Jensen and Maria Goeppert-Mayer in clarifying that there is a difference in the spin and the spin orbit configurations in the nuclei preferring in the one case the lower numbers of Eq. (8) and in the other case the higher numbers of Eq. (9).

We see now that the jump between the magic numbers 20 and 28 results systematically from the procedure of Table 1 without any need of a physical explanation of the spin etc. Vice versa one may find an explanation of the spin-orbit phenomenon related to the threefold multiplicity of Eq. (6) concluding that the stable nuclear shells are combined each with three quark links of the higher shell to one quark in the lower shell. For nuclei where there is a low saturation of these links, the nuclei have high spins and deviate strongly from spherical shape as is well known for nuclei of the minima in the SAD distribution (Fig. 1) between the maxima near the magic numbers. 
Table 1: Sequence $\mathrm{n}=0,1,2 \ldots$ of magic numbers with the values $\exp \left(Z_{n} / Z^{\prime}\right)$ and $R(n)=\exp \left[\left(Z_{n+1} Z_{n}\right) / Z^{\prime}\right]$ of Eq. (3) with $Z^{\prime}$ $=10$ from Eq. (1) as measured

\begin{tabular}{lllll}
\hline $\mathrm{N}$ & Magic number & $\operatorname{Exp}\left(\mathrm{Z} / \mathrm{Z}^{\prime}\right)$ & $\mathrm{R}(\mathrm{n})$ & $3 \mathrm{n}$ \\
\hline 0 & 2 & 1.221 & 1.882 & 1 \\
1 & 8 & 2.2225 & 3.321 & 3 \\
$2($ as n+1 in (3)) & 20 & 7.389 & ----- & ---- \\
$2(\operatorname{as~n}$ in (3)) & 28 & 12.1824 & 9.025 & 9 \\
3 & 50 & 148.413 & 24.53 & 27 \\
4 & 82 & 3640.95 & 81.45 & 81 \\
5 & 126 & 296558.5 & & \\
\hline
\end{tabular}

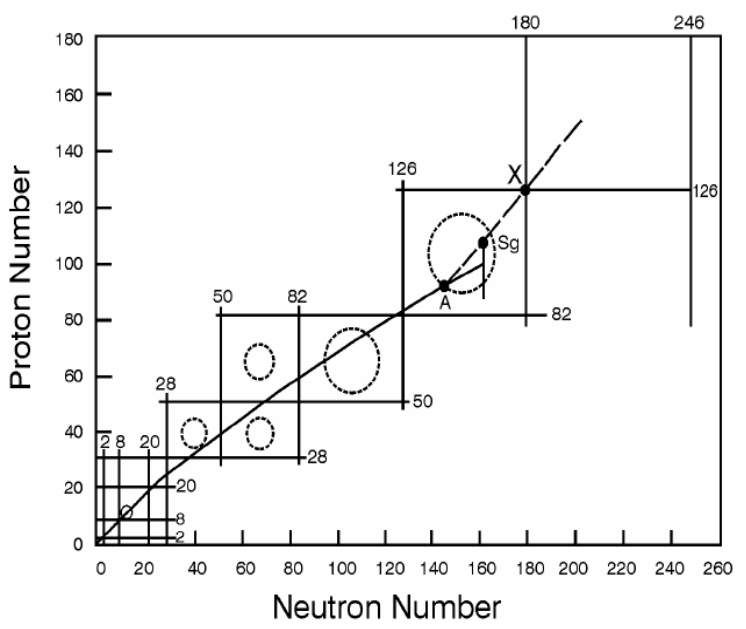

Fig. 3: Stable nuclides for various proton and neutron numbers up to about curium (point $\mathrm{A},{ }^{[3]}$ ) with extension $^{[7]}$ and standard magic numbers, Eq. (2) including new magic numbers Eq. (7).

A further conclusion relates to the higher magic numbers, Eq. (7), which differs very strongly from earlier concluded higher magic numbers ${ }^{[3]} 114,184$ and 228. Figure 3 shows the well-known trace of stable nuclides for various proton and neutron number up to about curium ${ }^{[5,6]}$. It is well known that from then on, the produced relative stable transuranium elements followed the dashed line with an increase of each proton with one neutron (and not two neutrons as below A). This line goes e.g., Through the well studied Seaborgium ${ }^{[7,8]}$ and crosses the nuclide ${ }^{126} \times{ }_{306}{ }^{[9]}$ which ideally agrees with our new magic number for the neutrons $\mathrm{N}=180=306-126$ while 126 is the wellknown number science Bagge ${ }^{[2]}$. According to (7), the nuclide ${ }^{126} \mathrm{X}_{306}{ }^{[9]}$ with double magic numbers is also an especially stable case derived from relativistic mean field Brueckner-Hartee-Fock calculations ${ }^{[6]}$.

\section{REFERENCES}

1. Audouze, J. And S. Vauclair, 1980. An Introduction to Nuclear Astrophysics, D. Reidel Publishing Comp. Dordrecht.

2. Bagge, E., 1948. Naturwissenschaften, 35: 376.

3. Brack, M., P. Quentin and D. Vautherin, 1978. Proceedings of the International Symposium on Superheavy Elements, Lubbock, Texas, 1978, Lodla, M.A.K., Edn., (Pergamon, New York) pp: 309. Sobieczewski, A., 1974 Phys. Sr. 10A, 47.

4. Haxel, O., J.H.D. Jensen and H.E. Suess, 1950. Zeitschr. f. Physik 128: 295.

5. Lefebvre, A., S. Bazookas, P. Agner, G. Bogaert, A. Coc, A. Denker, F.De Olivera, S. Forier, J. Gores, J. Kiener, J.M. Mason, M.G. Porquet, L. Rosier, V. Tatischeff, J.P Thibaud and M. Wiescher, 1997. Nuclear Physics A621, 199.

6. Rutz, K., M. Bender, T. Burvenich, T. Schilling, P.G. Reinhard, J.A. Maruhn and W. Greiner, 1997. Phys. Rev. C56, 238; Greiner, W., 1997. Nuovo Climento 110A, 1237.

7. Schadel, M., et al, 977. Nature 388,55; Schadel, M. and Kratz, J.V., 1997. Physikalische Blatter 53, 865; Armbruster, P., 1983. Proceedings International conference on Nuclear Phusics, Florence Italy; Blasi., P. And Picchi, R., 1984, Tipografia compositor, Bologna 343.

8. Wilets, L., 1987. Encyclopedia of Physical Sciences and Technology, Meyers A.A., Academic Press, New York, 9,300.

9. Hora, H., Miley, G.H. and Kelly, J.C., 2000. Trasactions Am. Nucl. Soc., 83,357. 